\title{
Chapter 4 \\ Resilience Engineering as a Quality Improvement Method in Healthcare
}

\author{
Janet E. Anderson, A. J. Ross, J. Back, M. Duncan and P. Jaye
}

\begin{abstract}
Current approaches to quality improvement rely on the identification of past problems through incident reporting and audits or the use of Lean principles to eliminate waste, to identify how to improve quality. In contrast, Resilience Engineering (RE) is based on insights from complexity science, and quality results from clinicians' ability to adapt safely to difficult situations, such as a surge in patient numbers, missing equipment or difficult unforeseen physiological problems. Progress in applying these insights to improve quality has been slow, despite the theoretical developments. In this chapter we describe a study in the Emergency Department of a large hospital in which we used RE principles to identify opportunities for quality improvement interventions. In depth observational fieldwork and interviews with clinicians were used to gather data about the key challenges faced, the misalignments between demand and capacity, adaptations that were required, and the four resilience abilities: responding, monitoring, anticipating and learning. Data were transcribed and used to write extended resilience narratives describing the work system. The narratives were analysed thematically using a combined deductive/inductive approach. A structured process was then used to identify potential interventions to improve quality. We describe one intervention to improve monitoring of patient flow and organisational learning about patient flow interventions. The approach we describe is challenging and requires close collaboration with clinicians to ensure accurate results. We found that using RE principles to improve quality is feasible and results in a focus on strengthening processes and supporting the challenges that clinicians face in their daily work.
\end{abstract}

J. E. Anderson ( $\varangle) \cdot$ J. Back · M. Duncan

Florence Nightingale Faculty of Nursing, Midwifery and Palliative Care,

King's College London, London, UK

e-mail: janet.anderson@kcl.ac.uk

A. J. Ross

Dental School, School of Medicine, University of Glasgow, Glasgow, Scotland, UK

P. Jaye

Guy's and St. Thomas' NHS Foundation Trust, London, UK

(C) The Author(s) 2019

S. Wiig and B. Fahlbruch (eds.), Exploring Resilience, SpringerBriefs

in Safety Management, https://doi.org/10.1007/978-3-030-03189-3_4 
Keywords Emergency department $\cdot$ Patient flow $\cdot$ Adaptive capacity $\cdot$ Quality improvement

\subsection{Context and Introduction}

Resilience Engineering (RE) is a new paradigm for conceptualising how work is accomplished in complex adaptive systems such as healthcare [1, 2]. It explicitly argues that the ability of organisations to adapt to pressures is what makes the system work, and is responsible for maintaining good outcomes in spite of problems and challenges. Workers are therefore seen as the key to creating safety, rather than being cast as the weak link in the system, prone to error and responsible for adverse outcomes. RE argues that it is the variability in the healthcare environment that drives the need for adaptation [3]. For example, surges in patient numbers, multiple patients deteriorating at the same time, lack of equipment and inappropriate staffing are all common variations in the conditions of work that require adaptation by workers. This way of thinking is different to the assumptions underpinning most quality improvement efforts that attempt to constrain human behaviour by specifying via protocol what actions should be taken [4], based on past problems identified through incident reporting, audits, or identification of waste through Lean principles.

These ideas appeal to clinicians and safety researchers because they reflect the reality of the messy clinical world in which conditions cannot always be anticipated and solutions have to be improvised. However, they need further interpretation and elaboration to move from a description of how work is achieved, to inform quality improvement [5]. RE is a theory about systems, and it needs to move beyond individual adaptations to consider how a system might support adaptive capacity. The four resilience abilities of responding, monitoring, anticipating and learning, proposed by Hollnagel [6], are promising and could provide a means for thinking about how adaptive capacity can be supported. For example, by considering whether and how a system learns it might be possible to devise ways to enhance learning and thereby increase quality. Despite these promising concepts, it is not immediately clear how to define the focus of an investigation based on RE since simply targeting learning in general, for example, seems unlikely to have a measurable effect on outcomes of interest.

In this research we developed a conceptual model to help us to think about how quality can be improved using insights from RE [4]. The CARe model proposes that variability in the healthcare environment often occurs because of a mismatch between demand and capacity. For example, a surge in patients is a problem if there are not enough staff rostered. Demand-capacity misalignments lead to adaptations in situ as staff attempt to work around problems to deliver care. Outcomes emerge from the interplay of misalignments and adaptations. A key insight from the model is that there are two potential routes to improving quality. Improvement efforts could focus on reducing misalignments between demand and capacity, thereby reducing the need for adaptations. This could potentially preserve resources that would otherwise be used to 
solve problems that have an obvious standardised solution (such as ensuring there is a good system for maintaining equipment) so that they could be used for coping with other less predictable problems. Alternatively, better support for adaptations and for strengthening the link between adaptations and good outcomes could also be a way to improve quality. Adaptations carry the risk that they will result in adverse outcomes because people are departing from protocol, or improvising solutions to problems not covered by the protocol, and may not be able to foresee all the implications of their actions. Supporting adaptation to ensure a good outcome is one goal of quality improvement from an RE perspective. For example, better systems for monitoring risk might be of use in enabling better planned adaptations when there are high risk conditions.

We have used RE theory and the CARe model to investigate whether RE can be used as a quality improvement method. Working longitudinally over several years, we have studied in depth the work systems the Emergency Department in a large London teaching hospital. The overall aims of the research were to use RE theory to develop and evaluate quality improvement interventions. To do this, we aimed to:

1. Build a deep and nuanced understanding of how work was achieved in the two units, including misalignments between demand and capacity and adaptations performed in situ;

2. Develop an interpretive process to identify interventions;

3. Design interventions with clinical teams and implement them;

4. Evaluate outcomes.

\subsection{Methodology}

For the ethnographic field work, two researchers, working as non-participant observers, first identified the main staff roles, processes, co-ordinating mechanisms, such as meetings and handovers, and technology and tools used. More focused observations were then conducted of the co-ordinating mechanisms and these included staff and team meetings, ward rounds, board rounds, patient flow meetings, and handovers. Finally, staff were shadowed as they carried out their everyday work and were asked to clarify decision making processes and reasons for actions. In depth interviews $(n=13)$ were also conducted with staff to probe for further detail about phenomena observed and clarify researchers' understanding of the observed work. Observational work occurred in both units concurrently.

Fieldwork data (104h of observation) were captured in written form and transcribed to electronic format. Field notes were expanded upon, combined with interview data, and then used as the basis for writing extended resilience narratives describing how outcomes emerge from the interplay of misalignments and adaptations. The aim was to describe trajectories of action that would serve as the basis for identifying opportunities for intervention. The resilience narratives were then analysed thematically using a combined deductive/inductive approach. Specifically, 
the analytic themes were - misalignments and pressures, variability, adjustments and adaptations, outcomes, goal trade-offs, anticipating, monitoring, responding and learning. The output of the analysis was a comprehensive description of the work system from the perspective of RE theory. At all stages of data collection and analysis we discussed and tested emerging findings within the research team, including clinicians, and with a clinical advisory group in each unit.

We then developed a structured collaborative process to design and implement interventions. The researchers subsequently developed a series of intervention proposals based on the ethnographic results. Clinical staff attended a series of workshops to discuss the results and advise on which interventions were most feasible and relevant. The design and implementation of the interventions was then conducted with the clinical partners who were most knowledgeable and influential in each unit.

\subsection{Results}

In the UK at the time the study was carried out, emergency departments were required to treat and discharge $95 \%$ of patients within four hours. Preventing breaches of this target was therefore a major focus of quality efforts. Regular patient flow meetings were held every two hours in the department, convened by a patient flow co-ordinator, to review patient numbers at all points in the department, flow through the department and to trouble shoot potential breaches of the waiting time target. Immediately before the meeting the patient flow co-ordinator would manually tally numbers of patients at various points in the department and verbally ascertain from clinicians which patients were likely to be imminently discharged and for those who were not, identify what was causing delay(s). Discussion at the meeting focused on how to address any particular problems and avert breaches, and often involved decisions to flexibly reallocate staff to different areas. Observations showed that each new meeting started with a new tally of patient numbers and did not refer to the actions recommended at the previous meeting two hours ago. Thus, it was not possible for staff to know;

- Whether the recommended action had been implemented;

- What the intended effect of the action was;

- What effect the action had in practice.

For staff reallocated to an area, it was not clear how long they were to remain and what they were trying to achieve. However, in some cases it was obvious. For example, if triage was overwhelmed with many simultaneous arrivals, a nurse flexed to this area would focus on reducing the numbers waiting. But there was no feedback to the flow co-ordinator and the next meeting would begin by reviewing numbers in each area with no reference to previous actions suggested.

In RE terms this resulted in an inability to monitor both the recommended action and its outcome, and an inability to learn from previous actions when convening the next two hourly meeting. The intervention that we developed involved redesigning the document used and the procedure for the meeting. The form was redesigned 
to enable capture of recommended actions and intended outcomes. The redesigned meeting process involved starting the meeting by reviewing actions from the previous meeting and evaluating whether they had had the desired effect. Decisions could then be made to address any problems that hadn't been solved in the previous meeting in a new way, before moving on to consider any additional problems that had developed in the previous two hours. These interventions aimed to increase the capacity of the patient flow meetings to monitor and learn from actions taken to improve patient flow in order to increase the adaptive capacity of the system.

\subsection{Discussion}

In this work we have demonstrated that RE can be used to identify opportunities to improve quality and to develop quality improvement interventions. In the rest of this section, we discuss some of the difficult issues and challenges faced in using RE to improve quality.

The intervention described here was designed to better support adaptive processes (adapting to patient inflow) and increase the likelihood that adaptations will lead to success (maintaining patient flow metrics). The method that we used focused attention on processes that could be strengthened to better support the challenges that clinicians had to resolve. Other quality improvement methods have different ways of identifying the targets of improvement efforts. For example, Lean approaches focus on identifying waste and intervening to reduce it and eliminate variation (for example $[7,8]$ ). Traditional quality improvement work often starts with reported adverse incidents which indicate that the system has produced unsuccessful outcomes [9]. However, targeting the causes of previous adverse incidents carries the risk of devising futile interventions for problems that would never occur again, and conversely, not addressing other system weaknesses that have yet to cause an adverse incident.

The process we developed was challenging, partly because it entailed an iterative sense making process involving interpretation using theory and observational data. For non clinicians it was challenging to understand all the nuances of the observations and clinical partners in the research team were crucial for ensuring that our interpretations and emerging results were accurate. The challenges included; steep learning curve for researchers; prolonged data collection time; effective analysis of a large amount of data; ensuring clinical engagement. However, many of these challenges apply to most qualitative health services research and are not insurmountable.

We did not start this study with an already identified quality problem that we wanted to solve. Instead, we used RE theory to understand in depth how the work system operated and where it could be strengthened. Nevertheless, the general approach used here could also be used to address a known problem, and indeed it may be much easier to achieve as the focus would be well defined from the outset. For example, improving medication errors in a hospital ward may be an appropriate aim of this approach. In this case, RE could provide a useful adjunct to existing quality improvement efforts by building a thorough understanding of work as done, misalignments 
between demand and capacity, sources of variability and the four resilience abilities in relation to medication administration. This would provide a thorough understanding on which to base the design of investigations and interventions. Without such a deep understanding of the system it may be difficult to design interventions that will be workable, sustainable and effective.

Evaluating quality improvement interventions based on RE is likely to be difficult. One challenge with evaluation is demonstrating that interventions increase resilience. Because adaptive capacity is expressed by a system in relation to a pressure or problem, we view it as an emergent property of the system rather than an outcome that can be measured [4]. For this reason we have not attempted to measure resilience. Our interventions have instead targeted the four resilience abilities (anticipating, monitoring, responding, learning) inferring that supporting these abilities will increase adaptive capacity. However, evaluating whether interventions have changed these abilities is also challenging and requires in depth qualitative work to understand the degree to which these abilities are affected. One concern is that interventions to strengthen processes are likely to be weakly linked to clinical outcomes and therefore it may be difficult to find strong evidence of effectiveness. This is a common problem in quality improvement that aims to change organisational processes $[10,11]$ and it can be particularly difficult to show that adverse incidents have been prevented.

\subsection{Further Development}

The approach that we have developed to quality improvement is resource intensive and required a well-grounded understanding of RE theory and practice. If this approach is to be useful in healthcare there is a need to produce guidance, streamline the process and more clearly articulate how to move from data collection to interpretation to intervention and evaluation. We are confident this can be done, but there is still a need to test the approach in a variety of settings. Primary care and mental health care are two settings in which this approach may be particularly valuable as both are less structured than acute care settings and rely to a greater extent on processes of social co-ordination and articulation that are even less amenable than acute care processes to standardisation and protocols.

\section{References}

1. E. Hollnagel, J. Braithwaite, R.L. Wears (eds.), Resilient Health Care (Ashgate, Farnham, 2013)

2. A. Ross, J.E. Anderson, Mobilizing resilience by monitoring the right things for the right people at the right time, in Resilient Health Care Volume 2: The Resilience of Everyday Clinical Work, ed. by R.L. Wears, E. Hollnagel, J. Braithwaite (Ashgate, Farnham, 2015), pp. 235-248 
3. R.L. Wears, E. Hollnagel, J. Braithwaite (eds.), Resilient Health Care Volume 2: The Resilience of Everyday Clinical Work (Ashgate, Farnham, 2015)

4. J.E. Anderson, A.J. Ross, J. Back, M. Duncan, P. Snell, K. Walsh, P. Jaye, Implementing resilience engineering for healthcare quality improvement using the CARE model: a feasibility study protocol. Pilot Feasibility Stud. 2(61) (2016)

5. J.E. Anderson, A.J. Ross, P. Jaye, Resilience engineering in healthcare: moving from epistemology to theory and practice, in Proceedings of the Fifth Resilience Engineering Symposium (2013). Resilience Engineering Association

6. E. Hollnagel, The four cornerstones of resilience engineering, in Resilience Engineering Perspectives, Volume 2: Preparation and Restoration, ed. by C.P. Nemeth, E. Hollnagel, S. Dekker (Ashgate, Farnham, 2009), p. 117-134

7. Z.J. Radnor, M. Holweg, J. Waring, Lean in healthcare: the unfilled promise? Soc. Sci. Med. 74(3), 364-371 (2012)

8. B.B. Poksinska, M. Fialkowska-Filipek, J. Engström, Does lean healthcare improve patient satisfaction? A mixed-method investigation into primary care. BMJ Qual. Saf. 26(2) (2016)

9. E. Hollnagel, Safety-I and Safety-II: The Past and Future of Safety Management (Ashgate, Farnham, 2014)

10. J. Øvretveit, D. Gustafson, Evaluation of quality improvement programmes. Qual. Saf. Health Care 11(3), 270-275 (2002)

11. J. Benn, S. Burnett, A. Parand, A. Pinto, S. Iskander, C. Vincent, Studying large-scale programmes to improve patient safety in whole care systems: challenges for research. Soc. Sci. Med. 69(12), 1767-1776 (2009)

Open Access This chapter is licensed under the terms of the Creative Commons Attribution 4.0 International License (http://creativecommons.org/licenses/by/4.0/), which permits use, sharing, adaptation, distribution and reproduction in any medium or format, as long as you give appropriate credit to the original author(s) and the source, provide a link to the Creative Commons license and indicate if changes were made.

The images or other third party material in this chapter are included in the chapter's Creative Commons license, unless indicated otherwise in a credit line to the material. If material is not included in the chapter's Creative Commons license and your intended use is not permitted by statutory regulation or exceeds the permitted use, you will need to obtain permission directly from the copyright holder.

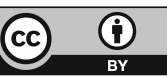

